\title{
THE SINGLE LIFE \\ OF RAPIDLY OSCILLATING AP STARS
}

\author{
S. HUBRIG \\ University of Potsdam \\ Am Neuen Palais 10, D-14469 Potsdam, Germany \\ N. KHARCHENKO \\ Main Astronomical Observatory \\ Golosiiv, 252650 Kiev - 22, Ukraine \\ AND \\ G. MATHYS \\ European Southern Observatory \\ Casilla 19001, Santiago 19, Chile
}

\section{Introduction}

Rapidly oscillating Ap (roAp) stars are cool magnetic Ap SrCrEu stars which pulsate in high-overtone $(n \gg l)$, low-degree $(\ell \leq 3) p$-modes, with periods from 6 to 15 minutes and typical amplitudes of a few millimagnitudes. 28 such stars are currently known. The roAp phenomenon is confined to a well-defined region of the Strömgren photometry parameter space (Martinez 1993). However, this region also contains other Ap stars, in which no pulsation could be detected, despite sometimes thorough searches. These apparently constant Ap stars (non-oscillating Ap stars, or noAp stars) appear remarkably similar to the roAp stars in many respects (e.g. colour indices, abundances, magnetic fields). Here we present recently found indications for differences between both groups.

\section{Kinematical Properties}

Recently, we have studied the kinematical properties of roAp and noAp stars by using new HIPPARCOS proper motions and parallaxes.

First, we have compared the positions in the H-R diagram of a sample of roAp stars and of a sample of noAp stars. Out of 28 roAp stars known, 14 were observed by HIPPARCOS. Our comparison sample of noAp stars contains 34 stars. We have selected all the Ap stars

- in which oscillations have been sought and have not been detected;

- whose photometric indices in the Strömgren system lie within the limits of occurrence of the roAp phenomenon (as defined by Martinez 1993); 
- for which HIPPARCOS data exist.

We have found that roAp stars, as a group, are $(-0.48 \pm 0.36)$ mag above the ZAMS, while the noAp stars are $(-1.30 \pm 0.64)$ mag above the ZAMS. Hence, in agreement with previous results (Mathys et al. 1996), roAp stars are found to be less luminous than their non-oscillating counterparts. This is also consistent with the recent result obtained by North et al. (1997). The difference in the absolute magnitudes between roAp and noAp stars found in this study is 0.82 magnitude. On the other hand, from the comparison of the kinematical characteristics (elements of the galactic orbits, total space velocities, and dispersion of the space velocities) calculated from HIPPARCOS data, we conclude that both groups are nearly identical, with some hints of older kinematics for roAp stars. This leads us to assume that roAp and noAp stars are of the same or only slightly different age, but that the roAp stars are less luminous, less massive, and less evolved (consistent with the results obtained by North et al. 1997). The difference between the masses of roAp stars and noAp stars is particularly important for the understanding of the origin of their oscillations. Plausibly, convection starts becoming efficient for the roAp stars. More generally, the difference of internal structure associated with the mass difference can probably explain why oscillations are observed only in the roAp stars.

\section{3. roAp stars versus noAp stars: binarity}

Until now, no roAp star is known to be a spectroscopic binary (SB). We obtained at least two measurements of the radial velocity of 14 of the 28 roAp stars, but we found no evidence for variation in any of these stars. The strong contrast with the rate of occurrence of binarity of $46 \%$ reported for cool Ap stars by Gerbaldi et al. (1985) is an indication that there is a real deficieny of binaries among roAp stars.

Relevant radial velocity data are scarce for noAp stars, due to the combination of their relative faintness (many have magnitudes between 8 and 10) and of the southern declination of most of them. However, all the noAp stars for which enough information is available (that is, 11 stars whose radial velocity has been repeatedly measured) either are SBs or show hints of binarity.

The interpretation of a difference in duplicity between roAp and noAp stars, and its meaning for the understanding of the origin of the pulsations in roAp stars are far from obvious. Even if the different internal structure of roAp stars is the reason for their pulsation, it is difficult to understand why no roAp star would be found to be a binary. That the latter is indeed true remains to be more definitely established, through additional observations. Such observations will help to establish which conditions must prevail for the appearance of rapid oscillations in cool Ap stars.

\section{References}

Gerbaldi, M., Floquet, M. and Hauck, B.: 1985, Astron. Astrophys. 146, 341

Martinez, P.: 1993, Ph. D. Thesis, University of Cape Town

Mathys, G., Kharchenko, N. and Hubrig, S.: 1996, Astron. Astrophys. 311, 90

North, P., Jaschek, C., Hauck, B., Figueras, F., Torra, J.-C., Mermilliod, J.-C. and Künzli, M.: 1997, ESA SP-402 (in press) 